\title{
Протологика и ее структура
}

\author{
В. И. ШАЛАК
}

ABStraCt. The article sets out a generalization of logical to the level of operating signs.

Keywords: protologic, semiotics, theory of signs, foundations of logic, combinatory logic, lambda-calculus

Основными структурами, вокруг которых строится большинство систем современной логики, являются предложения языка. В виде предложений формулируются законы науки. В виде множеств предложений формулируются научные теории. В виде операций преобразования предложений формулируются правила рассуждений.

Протологика [6] - это попытка обобщения понятия логического до уровня оперирования знаками. В качестве отправной точки, но не догмы для буквального повторения, выбрана теория знаков Ч. Пирса [5].

Основное понятие теории знаков - семиозис. Это ситуация, в которой некий обгект или явление (знак) служит для кого-то (для интерпретатора) представителем другого обвекта или явления (означаемое). Знаки не существуют сами по себе, а это мы наделяем их такой ролью. В зависимости от характера связи знака с означаемым Пирс делил их на три вида: индексы, иконы и символьи.

Индексъ связаны с означаемым непосредственно - причинными или пространственно-временными отношениями: дым костра, гром после молнии, запах мяса, буква $A$, указывающая на вершину треугольника, личные местоимения в ситуации их употребления и пр. Индексы всегда указывают на единичные объекты, и эти объекты действительно существуют. Согласно Пирсу, 
индексы - это знаки, посредством которых мы соприкасаемся с окружающей действительностью: «. . ни одно фактическое положение дел не может быть установлено без применения знака, служащего индексом» [5].

Иконы связаны с означаемым отношением сходства (наличием общего качества), которое может пониматься достаточно широко: фотографии, рисунки, скульптуры, диаграммы, графики, метафоры и пр. Пирс особо подчеркивает, что математика во многом построена на использовании иконических знаков и их преобразований. Примером могут быть формулы алгебраических преобразований. Заметим, что в равной мере это справедливо и для преобразований логических формул.

«. . Главное отличительное свойство иконы состоит в том, что посредством ее прямого наблюдения могут быть обнаружсен и другие истины, касающиеся ее обгекта, - истины, совершенно отличные от тех, которые были использованы при ее построении. . . А поскольку эта способность $к$ обнаружсению неожиданной истины и есть то, в чем состоит полезность алгебраической формуль, то иконический характер [в ней] будет преобладающим» [5].

В отличие от индексов, объект, представляемый иконой, может и не существовать в действительности (кентавр). Третий элемент семиозиса, означаемое, не является обязательным - могут существовать предметы или явления, которые мы наделяем ролью знаков, но которые не являются представителями чеголибо действительно существующего.

Cимволъ - это знаки в чистом виде. Они не связаны с означаемыми объектами ни в силу сходства, ни динамически, ни пространственно-временными отношениями. Они являются знаками лишь потому, что употреблены в этом качестве. Они существуют благодаря условному соглашению быть представителями других объектов.

В виде небольшого отступления от основной темы заметим, что, с нашей точки зрения, различие между индексами и иконами не столь велико, как кажется. И индексы, и иконы вторичны по отношению к означаемому. Они являются гомоморфными 
образами означаемого. Дым как знак-индекс костра - это всего лишь одна из многих характеристик сложного природного явления, которое мы называем костром. Остальные характеристики - пламя, температура, шум и пр. - «забыты», как и положено для результата гомоморфного отображения. Гром после молнии, запах мяса, положение флюгера тоже могут рассматриваться как гомоморфные образы реальных явлений. В случае иконических знаков сходство с означаемым - это прямое указание на их гомоморфное происхождение. Исходные иконические знаки, как и знаки-индексы, являются представителями реально существующих предметов и явлений. В случае Пегаса или Кентавра как графических изображений легко заметить, что это сложные знаки. Пегас сконструирован из двух иконических знаков - лошади и крыльев, а Кентавр - из лошади и головы человека. И лошадь, и крылья, и голова человека как графические изображения, являются гомоморфными образами реально существующих объектов, но в случае комбинирования они несовместимы между собой. В результате попытки совместить их в одном знаке получается сложный знак, не имеющий означаемого.

Свойством иконичности обладают все знаки. Чем больше в структуре знака содержится информации об означаемом, тем он более иконичен. Минимум иконичности у знаков, связанных с означаемым простой референцией, максимум иконичности у знаков, имеющих в качестве означаемого самих себя (такие тоже существуют).

Всякая знаковая система обладает внутренним синтаксисом, позволяющим из более простых знаков строить более сложные. Знание синтаксиса языка и связанных с ним семантических правил позволяет нам различать и понимать сложные выражения даже в том случае, если мы их никогда ранее не встречали.

«Язык представляет собой систему и единое иелое. Он организуется как упорядоченный набор различимых и служащих различению “знаков", которые обладают свойством разлагаться на единицы низшего порядка и соединяться в единицы более сложные. Эта большая структура, включающая в себя меньшие структуры нескольких уровней, и придает форму содер- 
жсания мысли. [...] Языковая форма является тем самым не только условием передачи мысли, но прежде всего условием ее реализации. Мъ постигаем мысль уже обормленной языковыми рамками» [2].

В общем случае об абстрактной знаковой системе мы знаем лишь то, что она имеет иерархическую структуру от простых знаков к сложным. При теоретическом изучении эту иерархию мы можем представлять, заключая группы знаков в скобки. В [6] было показано, что для этой цели нам достаточно всего одной пары скобок, которая позволяет из любых двух знаков $X$ и $Y$ построить более сложный знак $(X Y)$.

Необходимо предостеречь от упрощенного понимания знаков и синтаксиса знаковых систем. Вывеска «Продукты» (знаксимвол) вместе с дверью (знак-индекс), к которой она прибита, образуют сложный знак, возможным означаемым которого является торговая точка, где продают продукты. Это элемент знакового синтаксиса нашей культуры, с которым связано конкретное семантическое правило. Открыв дверь, мы можем обнаружить за ней одни лишь горы мусора, а не торговую точку, которой уже нет. Это не означает, что мы неправильно проинтерпретировали сложный знак. Просто в данном конкретном случае он оказался лишенным означаемого. Пустота и непустота знаков во многом похожа на ложность и истинность предложений, но имеет более общий характер.

Знаковые системы не являются статичными образованиями, а обладают свойством роста. Мы можем вводить в знаковые системы новые знаки-символы путем принятия соглашения об их взаимозаменимости с другими знаками. В терминах современной логики, знаки-символы - это результат операции принятия определений.

Многие виды интеллектуальной деятельности могут быть представлены как оперирование знаками, переход от одних знаков к другим. Это и доказательство теорем математики, и построение чертежей технических устройств, и написание музыкальных произведений. 
«... Человеческая иивилизачия невозможна без знаков и знаковых систем, человеческий разум неотделим от функиионирования знаков - а возможсно, и вообще интеллект следует отождествить именно с функиионированием знаков» [3].

Некоторые операции со знаками могут получать обоснование путем указания на свойства и связи между объектами, которые они представляют. Другие операции могут быть обоснованы исключительно внутренними свойствами знаковых систем, которые независимы от характеристик представляемой ими реальности. Именно последние операции и изучаются в протологике, поскольку они имеют универсальную применимость.

\section{Исходные символы}

1. Var - множество переменных;

2. Const - множество констант;

3. ), (- скобки.

Выражения этого языка будем называть термами. Они являются просто комбинациями переменных и констант, структурированными посредством скобок.

\section{Термы}

1. Если $x \in V a r$, то $x$ - терм;

2. Если $c \in$ Const, то $c$ - терм;

3. Если $X$ и $Y$ - термы, то $(X Y)$ - терм;

4. Ничто другое термом не является.

Терм вида $(X Y)$ не предполагает никакой подразумеваемой интерпретации, а всего лишь отражает на абстрактном уровне тот факт, что выражения языка структурированы синтаксическими правилами. 
Поскольку при нашем определении терма он может содержать много скобок, будем для облегчения восприятия опускать лишние скобки, предполагая их ассоциацию влево. Например, терм $((((X) Y) Z) U)$ после опускания скобок может быть записан просто как $X Y Z U$, а терм $((X(Y Z)) U)$ примет вид $X(Y Z) U$.

Определения. Если $T-$ терм, $F V(T) \subseteq\left\{x_{1}, \ldots, x_{n}\right\}, n \geqslant 0$ и $D$ - константа, то $\left(D x_{1} \ldots x_{n}\right)={ }_{\text {def }} T$ - определение.

$F V(T)$ служит обозначением для множества всех переменных, входящих в терм $T$.

Определение - это не выражение языка, а метаязыковое обозначение, фиксирующее некоторый интеллектуальный акт. Запись $\left(D x_{1} \ldots x_{n}\right)={ }_{\text {def }} T$ означает, что в объектном языке термы вида $\left(D x_{1} \ldots x_{n}\right)$ будут использоваться как сокращения для термов вида $T$.

В случае, когда $n>0$, определение является контекстуальным, а когда $n=0,-$ неконтекстуальным.

Каждое применение операции определения приводит к расширению множества констант языка и множества его термов. Чтобы отразить это, нам понадобится принять некоторые соглашения.

\section{Соглашения}

1. Пусть $\Delta-$ некоторое множество определений. Посредством $\operatorname{Const}(\Delta)$ будем обозначать множество всех констант, введенных в язык определениями $\Delta$, т.е. $\operatorname{Const}(\Delta)=$ $\left\{D:\left(D x_{1} \ldots x_{n}\right)=_{\text {def }} T \in \Delta\right\}$.

2. Посредством $L(\Delta)$ будем обозначать множество всех правильно построенных термов в языке с Const $=\operatorname{Const}(\Delta)$.

3. $T\left[T_{1} / x_{1}, \ldots, T_{n} / x_{n}\right]$ - результат одновременной подстановки термов $T_{1}, \ldots, T_{n}$ вместо переменных $x_{1}, \ldots, x_{n}$ в терме $T$.

Приняв соглашение, что $\left(D x_{1} \ldots x_{n}\right)$ является сокращением для $T$, мы приходим к правилу замены определяемого и определяющего. 
Правило замены. Если $\left(D x_{1} \ldots x_{n}\right)={ }_{d e f} T$ - определение, а $X\left\{D T_{1} \ldots T_{n}\right\}$ - терм, с выделенным конкретным вхождением терма $\left(D T_{1} \ldots T_{n}\right)$, то $X\left\{T\left[T_{1} / x_{1}, \ldots, T_{n} / x_{n}\right]\right\}$ есть результат замены $\left(D T_{1} \ldots T_{n}\right)$ согласно ранее принятому определению на $T\left[T_{1} / x_{1}, \ldots, T_{n} / x_{n}\right]$.

$$
(D E) \quad \frac{\left(D x_{1} \ldots x_{n}\right)=_{\text {def }} T \in \Delta, X\left\{D T_{1} \ldots T_{n}\right\}}{X\left\{T\left[T_{1} / x_{1}, \ldots, T_{n} / x_{n}\right]\right\}}
$$

При кажущейся сложности формулировки правила замены оно не представляет ничего необычного. Вспомним, как мы вводим в язык логики высказываний определение связки эквиваленции.

$$
p \leftrightarrow q=_{\text {def }}(p \supset q) \&(q \supset p)
$$

Встретив формулу $A \vee(B \leftrightarrow C)$ и пожелав устранить эквиваленцию в соответствии с принятым определением, мы должны подставить в дефиниенс вместо пропозициональной переменной $p$ формулу $B$, подставить вместо переменной $q$ формулу $C$, т.е. выполнить операцию одновременной подстановки $((p \supset q) \&(q \supset p))[B / p, C / q]$, получить в результате формулу $(B \supset C) \&(C \supset B)$ и после этого произвести замену $(B \leftrightarrow C)$ на $(B \supset C) \&(C \supset B)$. Результатом замены будет формула $A \vee((B \supset C) \&(C \supset B))$. Именно эта последовательность шагов и отражена в нашей формулировке правила замены.

Если константа $D$ введена контекстуальным определением $\left(D x_{1} \ldots x_{n}\right)=_{\text {def }} T$, где $n>0$, то применение правила замены $D E$ возможно лишь для тех ее вхождений, когда имеется требуемый контекст, т.е. $\left(D T_{1} \ldots T_{n}\right)$.

\section{Рассуждения (неформальные комментарии)}

В протологике нет теорем, но есть рассуждения, которые имеют довольно свободную форму при минимуме ограничений. 
Во-первых, в рассуждении мы можем использовать любую исходную константу языка из множества Const, поскольку предполагается, что в каждой возможной модели им приписана фиксированная интерпретация. Содержательно их можно понимать как исходные знаки-индексы и исходные иконические знаки, представляющие реально существующие объекты и явления.

Bo-вторых, если в рассуждении присутствуют два терма $X$ и $Y$, то по правилам синтаксиса мы можем объединить их в сложный терм $(X Y)$ и добавить на следующем шаге рассуждения. Обоснованием данного шага служит то, что правилам синтаксиса всегда соответствуют некоторые семантические правила, которые связывают значение (смысл) сложного знака со значениями (смыслами) составляющих его более простых знаков. Не исключено, что в результате этого сложный знак уже не будет представлять реально существующих объектов, но смысл у него все равно будет. Например, мы объединяем в один сложный знак голову человека и туловище лошади, получая Пегаса. Смысл этого знака очевиден каждому, хотя означаемое отсутствует. Подобным образом когда-то вводили в употребление отрицательные числа и корень из -1. Сначала многие протестовали, но потом оказалось, что новые знаки настолько удобны, что вместо отказа от их использования расширили само понятие числа, введя отрицательные комплексные числа.

B-mpeтъих, в любой момент рассуждения мы можем принять новое определение $\left(D x_{1} \ldots x_{n}\right)={ }_{d e f} T$, приводящее к расширению множества констант Const. Благодаря принятому определению, константа $D$ получает строгий смысл и может быть включена в рассуждение. В зависимости от конкретной знаковой системы она может получить, например, интерпретацию как кода (программы) построения терма $T$ из переменных $x_{1}, \ldots, x_{n}$ или имя гипостазированной структуры терма T. В реальной практике мы могли бы, например, определить предикатор Красивая_Девушка(x), а затем ввести в рассуждение термин Kрасота_Девушки. То есть предикат Красивая_Девушка $(x)$ можно представлять как контекст (Красота_Девушки, х), состоящий из имени гипостазы Kpacoma_Девушки и переменНой $x$. 
$B$-четвертых, если в арсенале принятых определений есть $\left(D x_{1} \ldots x_{n}\right)=_{\text {def }} T$, мы имеем полное право, когда это возможно, применять правило замены $D E$.

Рассуждением будем называть последовательность термов $<T_{1}, \ldots, T_{n}, \ldots>(n>0)$, каждый из членов которой удовлетворяет одному из следующих условий:

1. $T_{i} \in$ Const;

2. $T_{i}$ графически равен $\left(T_{g} T_{h}\right)$, где $g, h<i$;

3. $T_{i}$ введен на основании принятия определения $\left(T_{i} x_{1} \ldots x_{n}\right)={ }_{\text {def }} T$

4. $T_{i}$ получен по правилу $D E$ из терма $T_{h}$, где $h<i$.

В случае принятия определения новая определяемая константа добавляется к множеству констант языка Const $\cup\{D\}$, pacширяется множество определений $\Delta \cup\left\{\left(D x_{1} \ldots x_{n}\right)={ }_{\operatorname{def}} T\right\}$ и расширяется множество правильно построенных термов $L(\Delta \cup$ $\left.\left\{\left(D x_{1} \ldots x_{n}\right)=_{d e f} T\right\}\right)$. Два рассуждения могут оказаться идентичными как последовательности термов, но различаться определениями, посредством которых вводились новые константы. Эти рассуждения будут считаться разными.

Очевидно, что всякое рассуждение может быть продолжено многими способами. Поэтому множество всех рассуждений можно представить в виде дерева, все ветви которого потенциально бесконечны. Это дерево рассуждений мы и будем называть $\boldsymbol{n p o -}$ тологикой.

\section{Вопросы эффективности}

Очевидно, что множество всех рассуждений не является счетным, поскольку соответствующее ему дерево бесконечно ветвится в каждом своем узле, а мы знаем, что даже дерево последовательностей в языке из двух символов 0 и 1 с бинарным ветвлением континуально. Отсюда следует, что протологика не может 
быть задана эффективной процедурой. Строгое доказательство этого можно провести диагональным методом Кантора.

Поскольку рассуждения потенциально бесконечны, то они не могут быть предъявлены целиком, но вполне могут быть аппроксимируемы своими начальными фрагментами. Будем говорить, что рассуждение $R$ эффективно, если существует функция, которая для произвольного $n \in \mathbf{N}$ порождает первые $n$ членов $R(n)=<X_{1}, \ldots, X_{n}>$.

Допустим, что протологика эффективна. Согласно нашим определениям, это означает, что существует такая функция от двух аргументов $P(x, y)$, что $P(m, n)=R_{m}(n)$, т.е. эта функция порождает первые $n$ членов $m$-го рассуждения.

Для опровержения гипотезы об эффективности протологики достаточно эффективно определить такое рассуждение $S$, что $S(n) \neq R_{n}(n)$. Это сделать совсем несложно. Можно воспользоваться правилом добавления новых символов, которое не детерминировано предыдущими шагами рассуждения. Поскольку функция $S$ определена эффективно, то она должна совпадать с одной из функций $R_{k}$. Но по построению $S(k) \neq R_{k}(k)$. Мы получили противоречие, которое позволяет заключить, что протологика не может быть задана эффективно.

\section{Заключительные замечания}

Прежде чем отвечать на вопрос, хорошо или плохо то, что протологика не может быть задана эффективно, необходимо ответить на другой. В чем смысл протологики? В обычных дедуктивных логических системах одним из центральных является понятие вывода формулы из посылок. Вывод определяется как непустая конечная последовательность формул, построенная по фиксированным правилам. Отсюда естественным образом возникает задача поиска вывода. В протологике ничего подобного нет. В протологике любой терм языка выводим линейно за конечное число шагов, и задача поиска вывода просто не имеет смысла, поскольку решается тривиально. Терм просто собирается из подтермов. Основная цель протологического рассуждения заключается не в этом, а во введении в язык посредством определений новых символов, которые обладали бы полезными 
свойствами. Что такое полезное свойство? Это понятие вряд ли возможно определить строгим образом. Полезные свойства новых символов обнаруживаются лишь тогда, когда ими начинают оперировать, когда они включаются в познавательную практику. Протологика по своей природе просто не может быть эффективной, так как ее основной принцип - найди то, не знаю что, но чтобы это было полезно. Лишь после введения в язык новых символов и исследования их свойств, становится понятно, полезны они или нет. Поясним сказанное на конкретном примере из области геометрии.

Вряд ли можно сомневаться в том, что понятие треугольника полезно в геометрии. Но это выясняется лишь после того, как при анализе данного понятия мы обнаруживаем в нем очень много зависимостей, связывающих между собой различные его внутренние и внешние характеристики: стороны, углы, площади, отношения подобия, триангуляция многоугольников и пр. Евклид в «Началах» [4] после принятия ряда определений и аксиом приступает к доказательству теорем о свойствах треугольников. Первая теорема посвящена возможности построения по данному отрезку равностороннего треугольника. Затем он переходит к доказательству более сложных теорем. Это и есть исследование полезных свойств понятия треугольника, но оно стало возможным лишь после того, как было введено в язык.

В рассуждениях протологики мы можем выделить синтетические шаги, совершаемые при построении новых термов, и аналитические, в ходе которых исследуются свойства, которыми эти термы обладают. Как уже было сказано, в протологике мы можем построить и включить в рассуждение любой терм, удовлетворяющий синтаксису знаковой системы. Это синтетические шаги рассуждения. За ними могут следовать аналитические шаги, целью которых является исследование полезных свойств построенного терма. Закономерно возникает следующий вопрос. Коль скоро может быть построен любой терм, то не проще ли вообще опустить синтетическую часть рассуждений и исследовать лишь аналитическую? Обратимся еще раз к Евклиду. В первой теореме по данному отрезку он строит две окружности, находит точку их пересечения и соединяет ее с концами исходного 
отрезка. Затем следует доказательство, что полученная фигура является равносторонним треугольником. Как видим, синтетическая и аналитическая части рассуждения четко разграничены. Но вторая часть доказательства невозможна без обращения к первой. По полученной в результате фигуре нельзя определить, что это равносторонний треугольник, если не обратиться к шагам его построения, что и делает Евклид. Анализ невозможен без знания структуры того, что анализируется, а структура возникает на этапе синтеза. Многие последующие теоремы имеют аналитический вид, но опираются на то, что анализируемые фигуры (термы) были построены (синтезированы) и исследованы (проанализированы) в ранее доказанных теоремах.

В [6] для аналитической части протологических рассуждений был использован термин логика дефинициальной дедукиии. Она теснейшим образом связана с такими логико-математическими формализмами, как комбинаторная логика Шейнфинкеля Карри $[8,10]$ и $\lambda$-исчисление Чёрча $[9,1]$. В [6] было показано, что средствами протологики могут быть определены все функции двузначной булевой алгебры. Более того, в ней могут быть определены все конечнозначные табличные функции. И, наконец, в ней можно определить все рекурсивные функции. Необходимо заметить, что для этого нет необходимости делать какие-то предположения о предметной области, о которой мы рассуждаем. Все функции определимы внутри языка и потому проецируются на любую предметную область совершенно независимо от ее специфики. В терминологии Канта вычислимые функции должны быть названы априорными. Это же справедливо и для того фрагмента математических знаний, который может быть представлен протологикой. Интересно исследовать его границы.

Существует несколько моделей развития науки. $\mathrm{K}$ их числу можно добавить и протологическую. В первом приближении можно считать, что состояние науки в какой-то момент времени характеризуется тем понятийным аппаратом, который в ней используется. В терминах протологики это может быть представлено набором принятых определений на конечном удалении от корня дерева. Дальнейшее развитие может быть представле- 
но как исследование ветвей дерева, выходящих из данного узла, как попытки продолжить строить дерево протологики. Если одно из продолжений оказывается удачным, то наука делает следующий шаг в своем развитии, все более удаляясь от истоков. Это кумулятивное развитие. Но может оказаться, что уже принятая система определений не слишком удачна. В этом случае следующий шаг в развитии науки заключается не в продолжении построенной ветви дерева, а в отходе на несколько шагов к корню дерева для последующего роста в другом направлении. Такой шаг в построении дерева протологики может быть истолкован как отказ от прежней парадигмы и принятие новой.

Легко заметить, что протологика не соприкасается с опытом. Отсюда возникает вопрос, каким образом с помощью протологики могут быть представлены теории, толчком для возникновения которых служат эмпирические наблюдения? Это можно сделать путем расширения набора правил построения рассуждений. То есть на некотором шаге построения одного из рассуждений протологики (одной из ветвей дерева) мы начинаем использовать новые правила добавления знаков, которые не могут быть обоснованы внутренними свойствами знаковой системы. Это и будет означать переход к построению протологической теории. Геометрия Евклида может быть представлена как неоконченное протологическое рассуждение, самыми первыми шагами которого были принятия определений и введение в рассмотрение новых знаков-символов. Первые три постулата Евклида - это частные случаи правила перехода от термов $X$ и $Y$ к терму $(X Y)$, а пятый постулат означает переход к протологической теории, поскольку в нем постулируется, но никак не обосновывается существование точки пересечения двух прямых. Это объясняет, почему именно вокруг него и разыгрались последующие баталии.

\section{Литература}

[1] Барендрегт X. Ламбда-исчисление. Его синтаксис и семантика. М.: Мир, 1985. 606 с. 
[2] Бенвенист Э. Категории мысли и категории языка // Общая лингвистика. Благовещенск: БГК им. И.А. Бодуэна де Куртенэ, 1998. C. 104-114.

[3] Моррис Ч.У. Основания теории знаков // Семиотика. Том 1. Благовещенск: БГК им. И.А. Бодуэна де Куртенэ, 1998. С. 36-88.

[4] Начала Евклида. Книги I-VI. ОГИЗ, Москва-Ленинград, 1948.

[5] Пирс Ч.С. Избранные философские произведения. Пер. с англ. М.: Логос, 2000.

[6] Шалак В.И. Протологика: новый взгляд на природу логического // Дисс... . докт. филос. наук. М., 2010.

[7] Шалак В.И. Логический анализ дефинициальной дедукции // Логические исследования. Вып.15. М.: Наука. С. 266-283.

[8] Шейнфинкель М.И. О кирпичах математической логики // Логические исследования. Вып.15. М.: Наука. С. 232-246.

[9] Church A. The Calculi of Lambda-Conversion // Annals of Mathematical Studies. 1941, № 6.82 p.

[10] Curry H.B., Feys R. Combinatory Logic. V.1. Amsterdam, 1958.

[11] Shalack V.I. Semiotic foundations of logic // Logical Investigation. 2013. Issue 19. P. 225-237.

\section{References (transliteration)}

[1] Barendregt H. Lambda-ischislenie. Ego sintaksis i semantika. M.: Mir, 1985.

[2] Benvenist E. Kategorii mysli i kategorii jazyka // Obshhaja lingvistika. Blagoveshhensk: BGK im. I.A. Boduena de Kurtene. 1998. S. 104-114.

[3] Morris Ch.U. Osnovanija teorii znakov // Semiotika. Vol. 1. Blagoveshhensk: BGK im. I.A. Boduena de Kurtene. 1998. S. 36-88.

[4] Nachala Evklida. Knigi I-VI. OGIZ, Moskva-Leningrad, 1948.

[5] Pirs Ch.S. Izbrannye filosofskie proizvedenija. Per. s angl. M.: Logos, 2000.

[6] Shalak V.I. Protologika: novyj vzgljad na prirodu logicheskogo // Diss... . dokt. filos. nauk. M., 2010.

[7] Shalak V.I. Logicheskij analiz definicial'noj dedukcii // Logicheskie issledovanija. 2009. Vyp. 15. M.: Nauka. S. 266-283.

[8] Shejnfinkel' M.I. O kirpichah matematicheskoj logiki // Logicheskie issledovanija. 2009. Vyp. 15. M.: Nauka. S. 232-246.

[9] Church A. The Calculi of Lambda-Conversion // Annals of Mathematical Studies. 1941.№ 6.82 p. 
[10] Curry H.B., Feys R. Combinatory Logic. V.1. Amsterdam. 1958.

[11] Shalack V.I. Semiotic foundations of logic // Logical Investigation. 2013. Issue 19. P. 225-237. 